\section{Geschlechtsverkehr bringt E. coli daher}

\author{
Nach ungeschütztem Vaginalverkehr kann es zu einer Kolonisation der Vagina \\ mit E. coli kommen. Dafür fanden sich Ursachen unterschiedlicher Art.
}

$\mathrm{D}$ er weibliche Genitaltrakt verfügt über verschiedene natürliche $\mathrm{Ab}$ wehrmechanismen gegen bakterielle und virale Erreger. Ungeschützter Geschlechtsverkehr erhöht bekanntlich das Risiko für eine vaginale Kolonisation mit E. coli. Studien haben gezeigt, dass das weibliche Genitalsekret sexuell abstinenter Frauen eine Aktivität gegen E. coli, Herpes-simplex-Virus-Typ 2 (HSV2) sowie HIV aufweist. Bedingt wird diese natürliche Abwehr durch Zytokine, Chemokine, antimikrobielle Peptide, Antikörper sowie durch sezernierte Proteine der vaginalen Residualflora.

In einer Studie wurde jetzt der Hypothese nachgegangen, welche Auswirkungen der Koitus auf immunologische Reaktionen und die antimikrobielle Aktivität der vaginalen Schleimhaut hat. Dafür wurde Zervikovaginallavage (CVL) von 17 Frauen, die vor Studienbeginn 72 Stunden abstinent sein mussten, vor dem Geschlechtsverkehr sowie 2-6 Stunden und 10-14 Stunden postkoital untersucht. Zudem wurde zwischen vaginalem Koitus mit und ohne Kondom differenziert. Der $\mathrm{pH}$-Wert betrug vor ungeschütztem Koitus 4,6 (4,2-6,0), 2-6 Stunden postkoital 5,5 $(4,4-7,5)$ und 10-14 Stunden postkoital 4,9 $(4,3-6,8)$. Bei Kondomgebrauch änderte sich der $\mathrm{pH}$ Wert nicht. Weiterhin zeigte sich ein Anstieg des transformierenden Wachstumsfaktors- $ß 1$, einem zu den Zytokinen gehörenden Signalmoleküls mit direkter antiinflammatorischer Wirkung. Dagegen nahmen die Konzentrationen von humanem Beta-Defensin (HBD)-2, HBD-3 sowie von Interleukin-8 nach ungeschütztem Koitus ab. Defensine können Bakterien abwehren und die virale Replikation hemmen. Bezogen auf den Ausgangswert verminderte sich die inhibitorische Aktivität der CVL gegen E. coli postkoital nach 2-6 Stunden und blieb auch nach 10-14 Stunden erniedrigt. Bei Kondombenutzung fand sich dagegen keine Veränderung. Unbeeinflusst blieb die inhibitorische Aktivität der CVL gegen HSV-2 und HIV sowohl nach

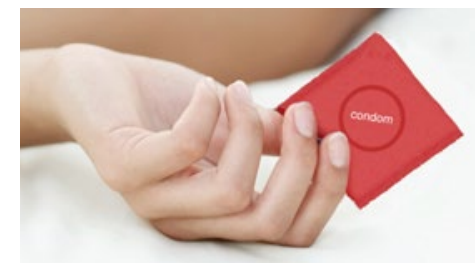

Kondome schützen - auch vor einer vaginalen Besiedlung mit $\mathrm{E}$ coli.

geschütztem als auch nach ungeschütztem Koitus. Untersucht wurde auch die Bedeutung von Sperma auf das Wachstum von E. coli. Gepoolte Samenflüssigkeit führte in vitro zu einer dosisabhängigen Vermehrung von E. coli. Wird Samenflüssigkeit zu CVL von sexuell abstinenten Frauen hinzugegeben, verringert sich dosisabhängig die inhibitorische Aktivität des Zervikovaginalsekrets.

Nakra NA et al. Loss of innate defense following unprotected vaginal sex. J Infect Dis 2015. first published online October 13, doi:10.1093/infdis/ jiv488

\section{Kommentar}

Die Studie liefert grundlegende neue Erkenntnisse zur Klärung der Frage, warum es zur vorübergehenden vaginalen Kolonisation von E. coli kommt. Als Ursache wurde neben der postkoitalen Veränderung des $\mathrm{pH}$-Werts auch eine Aktivitätsänderung mehrerer Zytokine und Chemokine gefunden. Auch erwies sich Sperma als Risikofaktor für die ansonsten vorhandene natürliche Aktivität des Zervikovaginalsekrets gegen E. coli. Frauen, die nach ungeschütztem Geschlechtsverkehr zum Beispiel unter rezidivierenden Harnwegsinfektionen leiden, könnten von den Ergebnissen dieser Studie zukünftig profitieren.

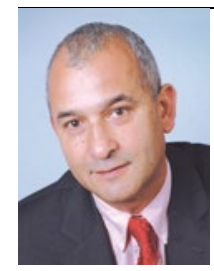

Prof. Dr. med. Tino F. Schwarz Zentrallabor, Stiftung Juliusspital Würzburg nach ungeschütztem Geschlechtsverkehr 